\title{
Elemente und Ephemeride des Kometen 1905 b.
}

Aus den Beobachtungen Bamberg Nov. I 8, Glasgow Mo. Nov. I 9 und Utrecht Nov. 20 habe jch mit Berücksichtigung der Korrektionen für Aberration und Parallaxe die folgenden Elemente abgeleitet :

$$
\left.\begin{array}{rl}
T & =1905 \text { Okt. } 27.4926 \text { Berlin } \\
\omega & =135^{\circ} 38.7 \\
\delta & =22345.4 \\
i & =13854.6 \\
\log q & =0.02626
\end{array}\right\} 1905.0
$$

Die Elemente lassen im mittleren Ort den nicht $z u$ beseitigenden Fehler von

$$
\Delta \lambda \cdot \cos \beta=+\mathrm{I}: \mathbf{1} \quad \Delta \beta=+\mathrm{I}: 6
$$

im Sinne $(B-R)$ übrig.*) Die Korrektion der Ephemeride beträgt am $2 \mathrm{I}$. November nach der umstehend angeführten

\begin{tabular}{|c|c|c|c|c|c|c|}
\hline 1905 & $\alpha$ & & $\delta$ & $\log r$ & $\log A$ & $\mathbf{H}$ \\
\hline Nov. 20 & $23^{\mathrm{h}} 54^{\mathrm{m}} 12^{\mathrm{s}}$ & $+61^{\circ}$ & $46: 5$ & $0.054^{8}$ & $9 \cdot 3788$ & 1.08 \\
\hline 2 I & $46 \quad 25$ & $5^{2}$ & 53.9 & 0571 & $386 \mathrm{I}$ & 1.03 \\
\hline 22 & 4 I 4 I & 44 & 29.2 & 0594 & $403 I$ & 0.94 \\
\hline 23 & $3^{8} \quad 32$ & $3^{6}$ & $5^{0.3}$ & 0617 & 4278 & 0.83 \\
\hline 24 & $3^{6} 20$ & $3^{\circ}$ & 5.4 & 0642 & 4576 & 0.72 \\
\hline 25 & $34 \quad 45$ & 24 & $15 \cdot 3$ & 0666 & 4904 & $0.6 \mathrm{I}$ \\
\hline 26 & 3335 & I 9 & 15.6 & 0692 & 5246 & 0.52 \\
\hline 27 & $\begin{array}{ll}32 & 43\end{array}$ & I 4 & 59.9 & 0717 & 5588 & 0.44 \\
\hline 28 & $\begin{array}{ll}32 & 4\end{array}$ & I I & 21.5 & 0.0744 & 9.5925 & 0.37 \\
\hline 29 & 3 I 34 & 8 & 14.0 & & & \\
\hline $3^{\circ}$ & 233 I I3 & +5 & 33.2 & 0.0797 & $9.65^{64}$ & 0.27 \\
\hline
\end{tabular}
Bamberger Beobachtung: $\Delta \alpha=-5^{\mathrm{s}}, \Delta \delta=+3: 2$. Die abweichenden Zahlen im Zirkular Nr. 80 der Zentralstelle

\begin{tabular}{|c|c|c|c|c|c|c|}
\hline 1905 & $\alpha$ & & $\delta$ & $\log r$ & $\log 4$ & $\mathbf{H}$ \\
\hline Nov. 30 & $23^{h} 3^{I^{\mathrm{In}}} 13^{s}$ & +5 & $33: 2$ & 0.0797 & 9.6564 & 0.27 \\
\hline Dez. I & $305^{8}$ & 3 & I $3 \cdot 3$ & & & \\
\hline 2 & 3049 & +1 & 11.8 & 0.0853 & 9.7150 & 0.20 \\
\hline 3 & 3044 & -0 & $35 \cdot 3$ & & & \\
\hline 4 & $3^{\circ} 4^{2}$ & 2 & 9.4 & 0.0910 & $9.768 \mathrm{I}$ & o.1 5 \\
\hline 5 & 3044 & 3 & 32.6 & & & \\
\hline 6 & 3049 & 4 & 46.6 & 0.0968 & $9.8 \times 63$ & 0.12 \\
\hline 7 & $3^{\circ} 5^{6}$ & 5 & 53.5 & & & \\
\hline 8 & $3 \times 5$ & 6 & 53.6 & 0.1027 & $9.860 \mathrm{I}$ & 0.09 \\
\hline 9 & 3116 & 7 & 47.9 & & & \\
\hline Io & $\begin{array}{lll}23 & 31 & 28\end{array}$ & -8 & $3^{6.1}$ & 0.1086 & 9.9002 & 0.08 \\
\hline
\end{tabular}
beruhen auf einer früheren, später von Prof. E. Hartwig berichtigten Mitteilung.

Ephemeride für I $^{\text {h }}$ M. Z. Berlin.

Kiel, Bureau der Astr. Nachrichten, I g05 Nov. 2.

*) Die Ursache liegt, wie sich leider erst nachträglich herausgestellt hat, an einem Fehler in der Bamberger Beobachtung von Nov. I8. Kr.

\section{Var. oder Nova 116.1905 Arietis.}

Auf zwei gestern nacht, I $9 \circ 5$ Nov. 6, zur Verfolgung der Planeten RX, RY und RZ aufgenommenen Platten findet sich ein Stern, ein wenig heller als I 2. Größe, der auf den Aufnahmen vom I. November noch nicht vorhanden war. Wir haben den Stern mit rechtwinkligen Koordinaten an die Sterne AG. Berlin A. 908 und $9 \times 8$ angeschlossen und den folgenden Ort erhalten:

$\alpha_{1900}=3^{\mathrm{h}} 19^{\mathrm{m}} \times 9^{\mathrm{s}} \cdot 29 \quad \delta$ × $900=+19^{\circ} 29^{\prime} 3^{\prime \prime} .7$.

Von dem Stern ist auf folgenden älteren Aufnahmen keine Spur vorhanden:

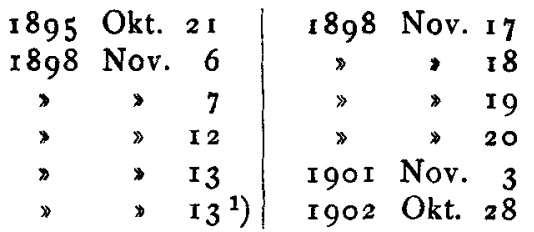

$$
\begin{aligned}
& 1902 \text { Nov. } 2 \text { I } \\
& \text { I 903 Aug. } 30 \\
& \text { - Okt. } 27 \\
& \gg \quad 27^{2} \text { ) } \\
& 1905 \text { Nov. I }
\end{aligned}
$$

Astrophysikalisches Institut Königstuhl-Heidelberg, I 905 Nov. 7. während er auf den nur 5 Tage späteren Aufnahmen vom 6. November als heller Stern abgebildet ist. Es ist deshalb

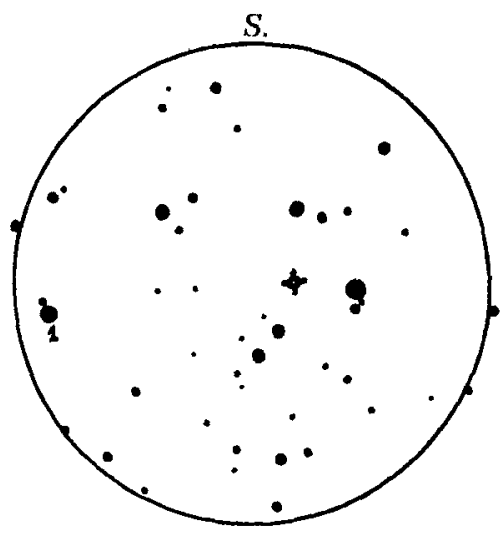
nicht unmöglich, daß er neu aufgetaucht ist.

Auf der nebenstehen. den, durch das Mikroskop gezeichneten Aufsuchungskarte wurde I Bogenminute 3 Millimeter groß gezeichnet. Der mit I bezeichnete Anschlußstern ist BD. + $99^{\circ} \cdot 524$. Er wird in der BD. mit $9.3 \mathrm{Gr}$. angegeben. Die schwächsten Sterne der Karte sind etwa $14.5 \mathrm{Gr}$.

M. und $G$. Wolf.

PS. vom 21. November. Der Stern 116.1905 Arietis ist rapide an Helligkeit heruntergegangen. Er hat jetzt nur noch die Größe ${ }^{2} 3 \cdot 5$ ca.

1) Vier Stunden später als die vorhergehende Aufnahme. $\left.{ }^{2}\right)$ Etwa anderthalb Stunden später als die vorhergehende Aufnalıme.

Inhalt zu Nr. 4056. M. Luizet. Observations et nouveaux éléments de l'étoile W Geminorum (Ch. 2335). 40I. - G. Abetti. (554) Peraga. 405 - M. und G. Wolf. Veränderliche um $\gamma$ Aquilae. 407. - Rambaud et $S y$. Observations de planètes et de comète. 409 - Beobachtungen des Kometen $1905 \mathrm{~b}$ (Telegramme). 4I3. - Elements and ephemeris of comet 1905 b. 413. - M. Ebell. Elemente und Ephemeride des Kometen 1905 b. 415. - M. und G. Wolf. Var. oder Nova Ir6.1905 Arietis. 415. 